\title{
Report of chronic myeloid leukemia in chronic phase from Ashirwad Hematology Centre, Mumbai, 2002-2009
}

\author{
M. B. Agarwal, \\ Usha M. Agarwal, \\ Shonali S. Rathi, \\ Sangeeta Masurkar, Bindi Zaveri \\ Department of Haematology- \\ Oncology, Ashirwad Haematology \\ Centre, Dadar, Mumbai, \\ Maharashtra, India
}

Address for correspondence:

Dr. M. B. Agarwal,

Ashirwad Haematology

Centre, Ghamat Lodge, 804/A,

Dr. B. Ambedkar Road, Dadar TT,

Mumbai - 400 014, Maharashtra,

India. E-mail: mbagarwal@

hotmail.com

\begin{abstract}
A B S T R A C T
We have analyzed our experience regarding use of tyrosine kinase inhibitors especially imatinib mesylate in patients of chronic myeloid leukemia-chronic phase over last 7 years at our center (2002-2009). The object was to report long-term efficacy (hematological, cytogenetic and molecular) and toxicity. Overall, 775 patients were treated. Out of these, 576 were analyzable with a median follow-up of 3.6 years. The median age was 42 years. Complete cytogenetic response (CCyR) was achieved in 351/576 patients, i.e., $62.1 \%$. Grade 3/4 adverse effects were observed in 36 patients, i.e., $6.25 \%$. Age under 40 years, low Sokal score, complete hematological response and CCyR were significant predictive factors for event free survival (EFS) on univariate analysis while low Sokal score and early chronic phase were significant predictive factors for EFS on multivariate analysis. Our results are almost similar to those reported from various studies from western population.
\end{abstract}

Key words: Chronic myeloid leukemia, cytogenetic response, imatinib mesylate, India, molecular response, toxicity

\section{INTRODUCTION}

Chronic myeloid leukemia (CML) is the most common leukemia in India as against chronic lymphocytic leukemia, which forms the most common leukemia in the western world. The age distribution of CML in India and in general, in Asian countries shows a shift to left with disease occurring in relatively younger subjects.

Although curable by allogeneic bone marrow transplantation (BMT), ${ }^{[1]}$ cost constrain and lack of matched donor availability and treatment related morbidity and mortality are major obstacles. In addition, the procedure is less widely available in the developing world including India. Treatment with interferon has become obsolete due to its inconvenience and toxicity. ${ }^{[2]}$ Older treatment like hydroxylurea and busulfan are no more preferred as these do not produce cytogenetic responses $(\mathrm{CyR})$.

\begin{tabular}{|l|l|}
\hline \multicolumn{2}{|c|}{ Access this article online } \\
\hline Quick Response Code: & Website: \\
\hline & Www.jmpo.org \\
\hline
\end{tabular}

Imatinib Mesylate (IM) is a potent and selective, competitive inhibitor of BCR-ABL protein. It is, at present, the frontline treatment for patients of CML-CP. The responses are high and durable. ${ }^{[3]}$ The quality-of-life is normal and there is substantial prolongation of survival. Its superiority over interferon has been established in IRIS study, which is now more than 8 years old.

We are reporting our long-term follow-up data from 848 consecutive patients of CML-CP seen at our center over last 7 years, i.e., 2002-2009, out of which 576 were analyzable with a median follow-up of 41 months, i.e., 3.4 years.

\section{MATERIALS AND METHODS}

This is a retrospective observational study. Patients were diagnosed to have CML-CP when the clinical and hematological picture was consistent and either $\mathrm{Ph}$ chromosome was documented by cytogenetic studies (Karyotyping) on marrow or BCR-ABL translocation was shown in peripheral blood by fluorescence in situ hybridization or polymerase chain reaction (FISH or PCR). A baseline history and physical examination was performed on all patients. Laboratory data included complete peripheral blood counts, hepatic and renal profile, lactic dehydrogenase and uric acid. Patients were divided into two 
groups: Early-chronic phase (ECP) and late-chronic phase (LCP) where LCP included patients who had received some or other modality of non-transplant-treatment of CML for a period of more than 6 months. All the responses (hematological, cytogenetic and molecular) were analyzed as defined in the literature.

IM was invariably obtained from Max Foundation through Glivec International Patients' Assistance Program (GIPAP), unless patient was not eligible or opted for a generic brand due to some other reason. The starting dose was invariably $400 \mathrm{mg}$ once a day orally, $1 \mathrm{~h}$ after dinner with a glass of water except for pediatric patients or those under the weight of $30 \mathrm{~kg}$, where $260 \mathrm{mg} / \mathrm{m}^{2}$ was the dose used (invariably rounded up to 200,300 or $400 \mathrm{mg}$ whichever closer to the calculated dose). The dose was reduced to $300 \mathrm{mg} /$ day in case of intolerance and it was escalated to 600 or $800 \mathrm{mg}$ in a step-wise fashion in those showing inadequate hematological, cytogenetic or molecular response (MR), i.e., failure to achieve a complete hematological response (CHR) by 12 weeks, complete cytogenetic response (CCyR) by 12 months and major molecular response (MMR) by 18 months. Patients were followed-up at monthly intervals to begin with and this included physical examination, complete blood counts and liver function tests. Cytogenetic analysis, i.e., karyotyping from marrow was ordered every 6 months until CCyR was achieved. Those unwilling to undergo marrow examination were followed-up by molecular testing (FISH in the early days and PCR during last 4 years) on peripheral blood at 3-6 monthly intervals. Adverse effects were documented at each visit and were graded.

Events were defined by the initial occurrence of any of the following:

1. Disease progression to accelerated phase (AP) or blast crises $(\mathrm{BC})$

2. Death due to any cause during treatment

3. Loss of CHR or major cytogenetic response

4. Intolerance to imatinib

Patients were censored at the time of imatinib discontinuation or if they were still receiving imatinib but had lost CyR.

CHR was defined as total leukocyte count (TLC) $<10,000 /$ $\mathrm{cmm}$ with less than $5 \%$ myelocytes and metamyelocytes, no blasts or promyelocytes in peripheral blood together with platelet count $<450,000 / \mathrm{cmm}$, no extramedullary disease and no evidence of AP or BC.

CyR were assessed on marrow samples by karyotyping using G-banding technique on at least 20 metaphases per sample. The response was said to be complete if there was no $\mathrm{Ph}$-positive metaphase, partial for 1-35\% $\mathrm{Ph}$-positive metaphases and major which included both complete and partial responses. MRs as stated earlier, were assessed by FISH in the early part of the study and by RQ PCR subsequently.

Event-free survival (EFS) was calculated from the date of the first IM dose to the first documentation of disease progression. Overall survival (OS) was calculated from the date of the first dose of IM to the date of last contact or death, whichever came first. Univariate and multivariate analysis was performed for factors predictive of a CCyR. Cox regression analysis was performed to evaluate following prognostic factors for survival: Age, gender, splenic enlargement, Hemoglobin $(\mathrm{Hb})<10 \mathrm{~g} / \mathrm{dl}$, TLC $>100,000 / \mathrm{cmm}$, platelet count $>450,000 / \mathrm{cmm}$, blasts $>5 /$ $\mathrm{cmm}$, basophils $>7 \%$, hematological and clinical response, CyR, Sokal score and previous treatment. A Sokal calculator was used to categorized patients into low, intermediate and high-risk groups.

Max Foundation has established GIPAP with the help of Novartis Pharmaceuticals globally including India. The primary objective of this program is to provide access to imatinib to as many patients as possible.

A total of 848 consecutive patients of CML-chronic phase were evaluated at our center between April 2002 and March 2009 (7 years). Out of these, 576 cases (68\%) are on imatinib at the moment, 73 cases $(9 \%)$ are on miscellaneous treatment while 199 cases $(23 \%)$ have either not followed-up with us (130 i.e., 15\%), expired (47 i.e., $5.5 \%)$ or discontinued imatinib due to adverse effects (22 i.e., $2.5 \%$ ). Out of patients receiving imatinib, 536/576 (93.1\%) are on Glivec through Max Foundation GIPAP program while the rest, i.e., $40(6.9 \%)$ are on generics. We noticed no difference in the efficacy and toxicity between Glivec and generics and hence the whole group has been advised together. Majority of patients receiving miscellaneous treatment are either on dasatinib (33 cases) or nilotinib (10 cases). Patients on dasatinib (21/33) were receiving it as a part of an international trial while those on nilotinib received the drug from Novartis on compassionate grounds. Six patients went on upfront BMT and two received alpha interferon.

Evaluation included EFS, OS, CCyR, MR and adverse events. Event-free and OSs were calculated by KaplanMeier estimates. Cox regression analysis was performed to evaluate 12 prognostic factors for survival. Univariate and multivariate analysis were performed for factors predictive of CCyR and MR. These included age, gender, splenomegaly, $\mathrm{Hb}<10 \mathrm{~g} / \mathrm{dl}$, TLC $>1,00,000 / \mathrm{cmm}$, platelet count $>4,50,000 / \mathrm{cmm}$, blasts $>5 / \mathrm{cmm}$, basophils $>7 \%$, 
Sokal score, CHR, CyR and patients presenting in ECP versus LCP.

\section{RESULTS}

Tables 1-3 give the age and sex distribution of the patients. Median age was 42 years. This is approximately a decade earlier than what is reported from the western world. There were 378 (65.6\%) males and 198 (34.4\%) females with M:F ratio of 1.9. This difference in sex distribution was probably due to more importance given to the male patients for treatment.

At the time of the present analysis, majority, i.e., 417 $(72.3 \%)$ were receiving the dose of $400 \mathrm{mg}$ once a day while $27(4.6 \%)$ were on $300 \mathrm{mg} /$ day, $74(12.8 \%)$ on $600 \mathrm{mg} /$ day and $58(10.3 \%)$ on $800 \mathrm{mg} /$ day. The reason for $300 \mathrm{mg} /$ day was usually intolerance to $400 \mathrm{mg} /$ day while the same for $600 \mathrm{mg}$ or $800 \mathrm{mg} /$ day was inadequate cytogenetic and/ or MR to $400 \mathrm{mg} /$ day. In all, 132 i.e. $23.1 \%$ of subjects received 600 or $800 \mathrm{mg}$ of IM daily. These details are shown in Table 4.

Median follow-up was 3.6 years with 158 (28\%) having followed for $6-8$ years and $340(59 \%)$ for between 3 and 6 years [Table 5$]$.

CCyR was achieved in $358(62.1 \%)$ patients. On multivariate analysis, low Sokal score $(P<0.0001)$ and ECP $(P<0.001)$ emerged as the most significant predictors for achieving CCyR. An estimated 6.2\% patients lost their CCyR. Grade III/IV toxicity was observed in $92(16 \%)$ of patients [Table 6].

Estimated 5-year EFS and OSs of these 576 were $72 \%$ and $87 \%$ respectively [Table 7]. On Cox regression analysis significant predictive factors for EFS were age $<40$ years $(P=0.005), \operatorname{CCyR}(P<0.0001)$, low Sokal score $(P=$ 0.005), complete clinical $(P<0.0001)$ and hematological responses $(P<0.0001)$ [Table 8]. Significant predictive factors for EFS on multivariate analysis were including low Sokal score and ECP [Table 9].

Tables 10 and 11 show patient characteristics according to CCyR.

Table 12 shows response to imatinib with respect to ECP or LCP. MR was assessed in lesser number of patients and this may explain the discrepancy between patients achieving CCyR and MMR.

Table 13 shows the hematological adverse events while Table 14 shows non-hematological adverse events.

\begin{tabular}{lcc}
\hline \multicolumn{3}{l}{ Table 1: Age distribution of patients with CML- } \\
CP $(\boldsymbol{n}=\mathbf{5 7 6})$ & & \\
\hline Age group (years) & Numbers & Percentage \\
$<10$ & 3 & 0.52 \\
$11-20$ & 17 & 2.95 \\
$21-30$ & 79 & 13.7 \\
$31-40$ & 148 & 25.6 \\
$41-50$ & 158 & 25.6 \\
$51-60$ & 109 & 18.9 \\
$61-70$ & 42 & 7.29 \\
$>71$ & 20 & 3.47 \\
\hline CML-Chronic myeloid leukemia; CP-Chronic phase
\end{tabular}

\begin{tabular}{lcc}
\hline \multicolumn{3}{l}{ Table 2: Age distribution of patients with CML- } \\
CP $(\boldsymbol{n}=\mathbf{5 7 6})$ & & \\
\hline Age group (years) & Numbers & Percentage \\
$<40$ & 247 & 42.8 \\
$>40$ & 329 & 57.2 \\
Total & 576 & 100 \\
\hline CML-Chronic myeloid leukemia; CP - Chronic phase
\end{tabular}

\begin{tabular}{lcc}
\hline Table 3: Sex distribution of patients with $\mathbf{C M L -}$ \\
CP $(\boldsymbol{n}=\mathbf{5 7 6})$ & \\
\hline Sex & Numbers & Percentage \\
Male & 378 & 65.6 \\
Female & 198 & 34.4 \\
Total & 576 & 100 \\
\hline
\end{tabular}

CML - Chronic myeloid leukemia; CP - Chronic phase

\begin{tabular}{|c|c|c|}
\hline Dose (mg) & Numbers & Percentage \\
\hline 400 & 417 & 72.3 \\
\hline $300 *$ & 27 & 4.6 \\
\hline $600 * *$ & 74 & 12.8 \\
\hline $800 * *$ & 58 & 10.3 \\
\hline Total & 576 & 100 \\
\hline
\end{tabular}

\begin{tabular}{lcc}
\hline \multicolumn{3}{l}{ Table 5: Follow-up duration* } \\
\hline Period (years) & Numbers & Percentage \\
$<3$ & 78 & 13.0 \\
$3-6$ & 340 & 59.0 \\
$6-8$ & 158 & 28.0 \\
Total & 576 & 100 \\
\hline *Median follow up: 3.6 years & &
\end{tabular}

DISCUSSION

At our center, during the period of 7 years, i.e., April, 2002-March, 2009, 848 cases were seen with CML-CP. Out of these, 576 patients were analyzable with respect to 


\begin{tabular}{lcc}
\hline \multicolumn{3}{l}{ Table 6: Results $(\boldsymbol{n}=\mathbf{5 7 6})$} \\
\hline Response & Numbers & Percentage \\
CHR & $545 / 576$ & 94.6 \\
CCyR & $358 / 576$ & $62.1^{*}$ \\
MMR & $21 / 292$ & 74.7 \\
Grade III/IV AE & 92 & 16.0 \\
\hline *22/351 subjects, i.e., $6.2 \%$ pts. lost CCyR; CHR - Complete hematological \\
response; Pts - Patients; CCyR - Complete cytogenetic response; MMR - Major \\
molecular response; AE - Adverse effect
\end{tabular}

\begin{tabular}{|c|c|c|}
\hline Estimated survival ( 5 years) & Numbers & Percentage \\
\hline EFS & $415 / 576$ & 72 \\
\hline OS & $501 / 576$ & 87 \\
\hline
\end{tabular}

\begin{tabular}{lc}
\hline $\begin{array}{l}\text { Table 8: Significant predictive factors } \\
\text { (on Cox regression analysis) }\end{array}$ \\
\hline Significant predictive factors & $P$ values \\
Age $<40$ years & 0.005 \\
CCyR & $<0.001$ \\
Low Sokal score & 0.005 \\
CHR & $<0.001$ \\
\hline
\end{tabular}

EFS - Event-free survival; CCyR - Complete cytogenetic response; CHR - Complete hematological response

\begin{tabular}{lc}
\hline $\begin{array}{l}\text { Table 9: Significant predictive factors for EFS } \\
\text { (on multivariate analysis) }\end{array}$ \\
\hline Significant predictive factors & $P$ values \\
Low Sokal score & $<0.0001$ \\
Early chronic phase & $<0.001$ \\
\hline Early chronic phase $-<6$ months treatment; EFS - Event-free survival
\end{tabular}

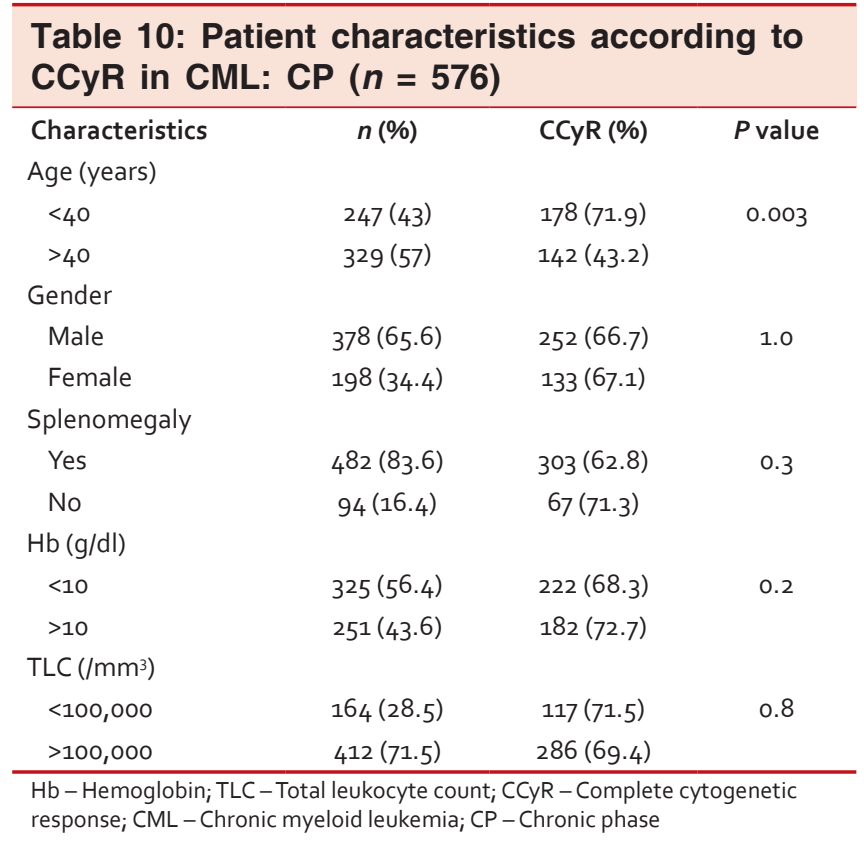

\begin{tabular}{lccc}
\hline \multicolumn{3}{l}{ Table 11: Patient characteristics according to } \\
CCyR in CML: CP $(\boldsymbol{n}=576)$ & & \\
\hline Characteristics & $n(\%)$ & CCyR (\%) & P value \\
Platelet count $\left(/ \mathrm{mm}^{3}\right)$ & & & \\
$<4,50,000$ & $433(75.2)$ & $310(71.5)$ & 0.01 \\
$>4,50,000$ & $143(24.8)$ & $75(52.7)$ & \\
Blasts in PB $\left(/ \mathrm{mm}^{3}\right)$ & & & \\
$<5$ & $512(88.9)$ & $350(68.3)$ & 0.005 \\
$>5$ & $64(11.1)$ & $25(39.2)$ & \\
Basophils in PB $\left(/ \mathrm{mm}^{3}\right)$ & & & \\
$<7$ & $560(97.3)$ & $372(66.5)$ & 0.5 \\
$>7$ & $16(2.7)$ & $10(62.5)$ & \\
Sokal score & & & \\
Low & $220(38.2)$ & $201(91.4)$ & $<0.0001$ \\
Intermediate & $222(38.6)$ & $136(61.2)$ & \\
High & $134(23.2)$ & $43(32.4)$ & \\
Phase of CML: CP & & & \\
ECP & $290(50.3)$ & $233(80.3)$ & $<0.0001$ \\
LCP & $286(49.7)$ & $142(49.7)$ & \\
\hline
\end{tabular}

CCyR - Complete cytogenetic response; CML - Chronic myeloid leukemia; CP - Chronic phase; PB - Peripheral blood; ECP - Early-chronic phase; LCP - Late-chronic phase

Table 12: Response to IM in CML-CP $(n=286)$ as per nature of chronic phase, i.e., early or late

\begin{tabular}{lcc} 
Response & ECP $(\boldsymbol{n}=\mathbf{2 9 0})$ & $\mathrm{LCP}(\boldsymbol{n}=\mathbf{2 8 6})$ \\
CHR & $281(96.9)$ & $264(92.3 \%)$ \\
Cytogenetic & & \\
CCyR & $233(80.3)$ & $125(43.7)$ \\
Major CyR & $254(87.6)$ & $160(56.0)$ \\
Minor/no CyR & $36(12.4)$ & $126(44.0)$ \\
Molecular $(n=292)$ & $(n=201)$ & $(n=91)$ \\
MMR & $149(74.1)$ & $69(75.8)$ \\
\hline
\end{tabular}

See text for explanation regarding discrepancy between CCyR and MMR. IM - Imatinib mesylate; CML - Chronic myeloid leukemia; $\mathrm{CP}$ - Chronic phase; CHR - Complete hematological response; CCyR - Complete cytogenetic response; CyR - Cytogenetic response; MMR - Major molecular response; ECP - Early-chronic phase; LCP - Late-chronic phase

\section{Table 13: Hematological AE in pts. with CML:} CP on IM $(n=576)$

\begin{tabular}{lcc}
\hline AE & \multicolumn{2}{c}{ No. of pts.(\%) } \\
\cline { 2 - 3 } Anemia & Grade I and II & Grade III and IV \\
Neutropenia & $156(25.3)$ & $14(2.4)$ \\
Thrombocytopenia & $33(5.7)$ & $6(1.0)$ \\
\hline CML -Chronic myeloid leukemia; CP - Chronic phase; AE - Adverse effect; \\
IM - Imatinib mesylate; Pts. - Patients
\end{tabular}

efficacy and toxicity of IM. The median age of patients was 42 years and the median follow-up was 3.6 years. CCyR, i.e., CCyR was achieved in 358/376 patients, i.e., $62.1 \%$. Grade $3 / 4$ toxicity was observed in 36 subjects, i.e., $6.25 \%$. Estimated 5-year EFS and OS were $72 \%$ and $87 \%$ respectively. On Cox regression analysis, age under 40 years, low Sokal score, CHR and CCyR were significant 


\begin{tabular}{|c|c|c|}
\hline \multirow[t]{2}{*}{$\mathrm{AE}$} & \multicolumn{2}{|c|}{ No. of pts. (\%) } \\
\hline & Grade I and II & Grade III and IV \\
\hline Diarrhea & $12(2.1)$ & $1(0.2)$ \\
\hline Musculoskeletal pain & $32(5.6)$ & $5(0.9)$ \\
\hline Nausea/vomiting & $26(4.5)$ & $11(1.9)$ \\
\hline Edema/weight gain & $47(8.2)$ & $32(5.6)$ \\
\hline Hypo-pigmentation & $108(18.8)$ & $11(1.9)$ \\
\hline Hepato-toxicity & 0 & $1(0.2)$ \\
\hline
\end{tabular}

predictive factors for EFS while on multivariate analysis, low Sokal score and ECP were the significant predictive factors for CCyR.

We conclude that long-term management of CML-CP with IM produces excellent and durable responses with the minimal toxicity. Our survival outcome is nearly similar to that reported from various western populations and superior to those reported from certain institutions in India. ${ }^{[4-8]}$ The difference is probably due to our center catering for higher socio-economic group (with higher education) coming from close vicinity permitting better follow-up.

\section{REFERENCES}

1. Gratwohl A, Brand R, Apperley J, Crawley C, Ruutu T, Corradini $\mathrm{P}$, et al. Allogeneic hematopoietic stem cell transplantation for chronic myeloid leukemia in Europe 2006:
Transplant activity, long-term data and current results. An analysis by the Chronic Leukemia Working Party of the European Group for Blood and Marrow Transplantation (EBMT). Haematologica 2006;91:513-21.

2. O'Brien SG, Guilhot F, Larson RA, Gathmann I, Baccarani M, Cervantes $\mathrm{F}$, et al. Imatinib compared with interferon and low-dose cytarabine for newly diagnosed chronic-phase chronic myeloid leukemia. N Engl J Med 2003;348:994-1004.

3. Druker BJ, Guilhot F, O'Brien SG, Gathmann I, Kantarjian H, Gattermann N, et al. Five-year follow-up of patients receiving imatinib for chronic myeloid leukemia. N Engl J Med 2006;355:2408-17.

4. Rosti G, Martinelli G, Bassi S, Amabile M, Trabacchi E, Giannini B, et al. Molecular response to imatinib in late chronic-phase chronic myeloid leukemia. Blood 2004;103:2284-90.

5. Zhao Y, Liu L, Wang Y, Wu G, Lai X, Cao W, et al. Efficacy and prognosis of chronic myeloid leukemia treated with imatinib mesylate in a Chinese population. Int $\mathrm{J}$ Hematol 2009;89:445-51.

6. Deshmukh C, Saikia T, Bakshi A, Amare-Kadam P, Baisane C, Parikh P. Imatinib mesylate in chronic myeloid leukemia: A prospective, single arm, non-randomized study. J Assoc Physicians India 2005;53:291-5.

7. Hay J, Bapsy PP, Babu KG, Loknatha. Imatinib mesylate in newly diagnosed patients with chronic myeloid leukaemia. ASCO annual meeting proceedings. J Clin Oncol 2007;25:17251.

8. Rajappa S, Varadpande L, Paul T, Jacob R, Digumarti R. Imatinib mesylate in early chronic phase chronic myeloid leukemia: Experience from a developing country. Leuk Lymphoma 2008;49:554-8.

How to cite this article: Agarwal MB, Agarwal UM, Rathi SS, Masurkar S, Zaveri B. Report of chronic myeloid leukemia in chronic phase from Ashirwad Hematology Centre, Mumbai, 2002-2009. Indian J Med Paediatr Oncol 2013;34:199-203.

Source of Support: Nil. Conflict of Interest: None declared. 